\title{
Das medidas de sustentabilidade às certificações sustentáveis: uma investigação sobre o desenvolvimento desses instrumentos
}

\section{Elcio Ricardo Leite Guimarães ${ }^{1}$ e Djalma Silva Guimarães Júnior ${ }^{2}$}

${ }^{1}$ RECIPREV - Saúde Recife. Av. Manoel Borba 488. Boa Vista. Recife-PE, Brasil (CEP 50070-000).E-mail: elcio.guimaraes@upe.br.

${ }^{2}$ Universidade de Pernambuco. Av. Eng. Abdias de Carvalho, 252. Torrões. Recife-PE, Brasil (CEP 50720-225).

Resumo. A sustentabilidade é uma temática que tem recebido cada vez mais a atenção da sociedade para o alcance do desenvolvimento ambiental, social e econômico, no sentido de se obter a prosperidade sem comprometer o direito à vida das gerações atuais e futuras. Este artigo faz um levantamento das principais medidas de sustentabilidade e de suas práticas através de certificações. Trata-se de uma contribuição para reunir e sistematizar informações sobre a evolução deste tema, seus indicadores e a sua prática nas organizações. Uma das motivações deste artigo é disponibilizar dados para o estudo sobre o uso das certificações de sustentabilidade, utilizando-se de levantamento de informações qualitativas, em ambientes aceitos pelo mundo acadêmico, através de documentos em publicações nacionais e internacionais, tendo por objetivo mapear as medidas de sustentabilidade e o seu desenvolvimento até as certificações sustentáveis. 0 estudo classifica-se como aplicado, exploratório e descritivo, realizado através de uma pesquisa documental e qualitativa. Foram identificados 153 textos que tratavam da temática, sendo parte deles selecionados para este trabalho. Conclui-se que a sociedade clama por maior celeridade aos agentes públicos, organizações e empresas no tocante à melhores e efetivas práticas de sustentabilidade, em função da imperiosa necessidade em se alcançar relações mais equilibradas entre os fatores sociais, econômicos e ambientais. Os avanços tecnológicos são parte da solução para se obter esse equilíbrio, através da criação de metodologias de composição e análise de indicadores e de outras tecnologias que possam ser universalizadas e tornarem-se um processo de uso comum no setor público e privado.

Palavras-chave: Medidas de sustentabilidade; Certificações; Selos verdes.

Abstract. From sustainability measures to sustainable certifications: An investigation into the development of this
Recebido

$15 / 06 / 2021$

Aceito

$29 / 08 / 2021$

Publicado

$31 / 08 / 2021$

Acesso aberto

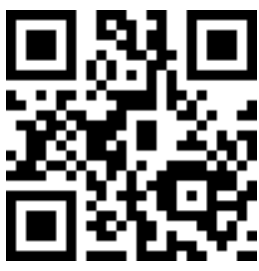

ORCID

(D) $0000-0002-1410-7744$

Elcio Ricardo Leite

Guimarães

(D) 0000-0002-4539-8492

Djalma Silva

Guimarães Júnior

ISSN 2359-1412/RBGAS-2021-0075/2021/8/19/38/1203

Rev. Bras. Gest. Amb. Sustent.

http://revista.ecogestaobrasil.net 
instruments. Sustainability is a theme that has received increasing attention from society for the achievement of environmental, social and economic development, in order to achieve prosperity without compromising the right to life for current and future generations. This article surveys the main sustainability measures and their practices through certifications. It is a contribution to gather and systematize information on the evolution of this theme, its indicators and its practice in organizations. One of the motivations of this article is to provide data for the study on the use of sustainability certifications, using a survey of qualitative information, in environments accepted by the academic world, through documents in national and international publications, in order to map the measures sustainability and its development to sustainable certifications. The study is classified as applied, exploratory and descriptive, carried out through documentary and qualitative research. 153 texts dealing with the theme were identified, part of which were selected for this work. It is concluded that society calls for greater speed to public agents, organizations and companies regarding the best and effective sustainability practices, due to the imperative need to achieve more balanced relations between social, economic and environmental factors. Technological advances are part of the solution to achieve this balance, through the creation of methodologies for the composition and analysis of indicators and other technologies that can be universalized and become a process in common use in the public and private sectors.

Keywords: Sustainability measures; Certifications; Green stamps.

\section{Introdução}

A história do desenvolvimento da humanidade tem propiciado um vasto conteúdo para o entendimento das relações sociais, econômicas e ambientais. Por diversas vezes, a busca por progresso ocasionou a exploração desenfreada dos recursos naturais e teve consequências.

De acordo com Roa e Vargas (2016), a saúde do ser humano está diretamente interligada com a questão ambiental, sendo fatores de efetiva ameaça os relacionados com o abastecimento de água potável e saneamento, o habitat em que vive, a alimentação, o uso de produtos químicos e os riscos ocupacionais dentre outros, porém, assim mesmo verifica-se pelo senso comum a ameaça em larga escala no mundo todo, advinda das variações climáticas, o esgotamento da camada de ozônio e a perda gradativa da biodiversidade.

Estudo realizado pela Organização Mundial da Saúde (OMS, 2002, 2003) registrou que a carga de doença, que significa a quantidade de anos de vida saudável, podem ser perdidos $24 \%$ deste tempo, devido à degradação do ambiente e, $23 \%$ de todas as mortes da humanidade poderiam ser atribuídas a causas ambientais, e estima-se que as mudanças climáticas sejam responsáveis por 250 mil mortes por ano entre 2030 e 2050. Impressionantemente, ainda, é o destaque de que $36 \%$ das mortes de crianças entre 0 e 14 anos tem sido atribuído a essas mesmas causas (OMS, 2002, 2003). De acordo com o Programa das Nações Unidas para o Desenvolvimento (PNUD, 2016), mais de 4 milhões de 
pessoas, em sua maioria nos países em desenvolvimento, sofrem problemas de saúde por contaminação com a água e $80 \%$ das enfermidades da população ocorrem por questões sanitárias em relação ao saneamento e à água.

Faz-se necessário destacar o péssimo momento atual da humanidade, em grave período de pandemia nos anos 2020 e seguintes, provocada pela COVID-19 e pelo novo coronavírus, onde só no Brasil, após um ano de enfrentamento desta situação, chega-se no mês de agosto de 2021 com cerca de 570 mil mortes, fato que certamente provocará alterações nos estudos e projeções realizadas pela Organização Mundial da Saúde (OMS).

Um dos principais entraves para o advento de um ambiente sustentável é a visão de curto prazo de boa parte de empresas, a qual tem desencadeado processos de desastres ecológicos, mesmo diante da possibilidade de afetar a sua própria sobrevivência no mercado, seus fornecedores, clientes, parceiros e a sociedade em geral (Dalmoro et al., 2009).

Em sendo a visão de curto prazo um entrave a ser destrinchado, dada a sua relevância no entendimento da problemática em relação à sustentabilidade, é preciso adentrar na temática do planejamento estratégico. De acordo com Lester et al. (2003) os executivos tendem a se preocupar apenas com as ameaças, que são problemas externos às organizações, do que com os fatores internos, nos primeiros anos de vida das suas existências, deixando de lado aspectos fundamentais como a sua estrutura e os elementos necessários para atingir a maturidade.

No estudo desenvolvido por Frezatti et al. (2010), sobre o perfil de planejamento e ciclo de vida organizacional nas empresas brasileiras, fica evidente a ausência de cultura para a prática do planejamento estruturado. Em uma abordagem com amostra de 111 empresas, de diversos segmentos, tamanhos e localidades, foram consideradas diversas fases do ciclo de vida organizacional, cruzando os dados com planejamento estratégico, orçamento e controle orçamentário. 0 estudo aponta que nas diversas fases do ciclo de vida das empresas pesquisadas, ficou caracterizada a insuficiente visão estratégica do planejamento.

Por outro lado, há sinais sociais trazendo uma nova realidade. Dentre as formas de pressão, indicando a necessidade de mudanças, pesquisas realizadas com consumidores tem detectado a crescente exigência no tocante às características de sustentabilidade das empresas, seus produtos e processos, a exemplo da divulgada por Kappa (2020) sobre ecommerce no período da pandemia, onde $35 \%$ dos consumidores não fariam compras online de uma empresa de moda se descobrissem que sua embalagem não era eco-friendly e destaca que $60 \%$ das mulheres valoriza as credenciais de sustentabilidade de uma marca refletidas em um pacote, enquanto no universo masculino são 51\% dos homens que valorizam essas credenciais (Embalagem Marca, 2020).

A IBM realizou pesquisa em parceria com National Retail Federation (NRF), em 28 países incluindo o Brasil, com 19 mil consumidores, onde se destaca que os consumidores estão priorizando as marcas de empresas que são sustentáveis, transparentes e mais alinhadas com os seus valores, estando dispostos a pagar mais e mudar seus hábitos de consumo para prestigiarem as marcas que sigam nessa direção. Em média, 70\% disseram ter disposição para pagar até $35 \%$ de valor adicional para empresas com produtos sustentáveis; $79 \%$ afirmaram ser importante que as marcas forneçam garantias de autenticidade, como certificações, ao oferecerem seus produtos (Newtrade, 2020).

De acordo com Kagermann e Wahister (2013), a perspectiva da inovação em sustentabilidade e na produção industrial, indica para mercados de trilhões de euros, dólares e outras moedas. Dentre esses mercados destacam-se o de Tecnologia e Engenharia de Máquinas, o de Energia Elétrica, o de Automação, o de Tecnologia da Informação, o de Investimentos em Pesquisa \& Desenvolvimento (P\&D), o de Internet das Coisas (IoT), o de Nuvens de Armazenamento e Transações, o de Robótica, o de Big Data e o de Ciber-físicos.

Rev. Bras. Gest. Amb. Sustent., 2021, vol. 8, n. 19, p. 1203-1217. 
Na Alemanha destaca-se a estratégia de segurança de dados, com programas de formação educacional, visando à inclusão digital e à melhoria da qualidade de vida, através de programas e projetos direcionados ao favorecimento da expansão da economia circular, onde os fatores da sustentabilidade são a tônica das ações desenvolvidas. 0 país exerce uma forte liderança no mercado da indústria manufatureira, notadamente na fabricação de máquinas e equipamentos. De acordo com o Ministério Alemão para a Cooperação com o Desenvolvimento, o Programa BMZ - 2030, é caracterizado por três fatores estratégicos, a boa governança, o respeito aos direitos humanos e a luta contra a corrupção. (Tatsachen Ueber Deutschland, 2021). Em 2019, a Alemanha quase atingiu a meta da ONU para investir $0,7 \%$ do PIB em cooperação ao desenvolvimento mundial, alcançando 0,6\% do PIB, com a contribuição de 23,81 bilhões de dólares, ocupando a segunda posição mundial enquanto doadora de recursos para programas de desenvolvimento sustentável. No setor de energia elétrica há aproximadamente 700 mil trabalhadores, dos quais 304.400 estão no setor de energias renováveis. Na área de mobilidade, os investimentos são massivos em pesquisa e inovação e em eletro mobilidade (Tatsachen Ueber Deutschland, 2021).

A China possui uma estratégia de ação na formação de clusters de desenvolvimento tecnológico com forte atuação ecológica, se destacando mundialmente na disputa pelo mercado de Tecnologia da Informação, Nuvem/Redes de Internet e Automação. Desde o seu 18ํㅡㄹ Congresso Nacional (PCCh), a China introduziu em seus programas várias grandes políticas incorporando o progresso ecológico em todas as áreas de desenvolvimento social, econômico, político e cultural. As políticas direcionadas ao equilíbrio ecológico foram divulgadas em linguagem comum, permitindo o aumento da consciência coletiva. A política ecológica compreende oito aspectos: direitos de propriedade de recursos naturais; exploração e proteção do espaço territorial nacional; planejamento do uso do solo; gestão e uso dos recursos naturais; uso oneroso dos recursos e políticas de compensação; governança ambiental; avaliação e aferição do progresso ecológico; e responsabilização e obrigações legais. Foi desenvolvido um novo padrão de fomento ao desenvolvimento com a criação de um índice de aferição da economia circular e do desenvolvimento verde das indústrias. Isso possibilitou que a China conquistasse a primeira posição mundial em capacidade instalada de geração de energia elétrica e solar. Ao final de 2015 o país atingiu a marca de $92 \%$ de esgoto urbano processado e $94,1 \%$ do lixo urbano processado com desintoxicação de material e reciclagem (China Hoje, 2021).

A Europa vem em segundo lugar no mercado de automação, destacando-se na criação e disponibilização de fundos internacionais em apoio a projetos de desenvolvimento sustentável. De acordo com o denominado Livro Branco lançado em 2017, documentado pela União Europeia para dotar os rumos das políticas públicas de sustentabilidade para seus países membros, com base nos parâmetros do Acordo de Paris sobre o Clima e para dar seguimento aos Objetivos de Desenvolvimento Sustentável (ODS), o continente Europeu demonstra a sua força de atuação em relação à sustentabilidade. A elaboração de um documento de reflexão pela Comissão Juncker, traz elementos centrados nos fundamentos políticos essenciais, nos fatores horizontais, que devem ser a base da transição para a sustentabilidade, focando nos aspectos educacionais, ciência, tecnologia, inovação, digitalização, finanças, preços, fiscalidade e concorrência, novos modelos empresariais, responsabilidade social, regras de comércio aberto, governança e coerência das políticas em todos os níveis (von der Leyen, 2019).

As seis prioridades definidas pela Comissão Juncker para o período 2019 e 2024 são (i) Pacto ecológico europeu: ser o primeiro continente com impacto neutro no clima; (ii) Uma Europa preparada para a era digital: capacitar as pessoas através de uma nova geração de tecnologias; (iii) Uma economia ao serviço das pessoas: criar um ambiente mais atrativo de investimento e um crescimento que crie empregos de qualidade para os 
jovens e para as pequenas empresas; (iv) Uma Europa mais forte no mundo: fazer a defesa do multilateralismo baseada em regras internacionais; (v) Promoção do modo de vida europeu: proteger o Estado de Direito para defender a justiça e os valores fundamentais da EU; e (vi) Um novo impulso para a democracia europeia: dar mais voz aos europeus e proteger a democracia de interferências externas tais como a desinformação e as mensagens de ódio (von der Leyen, 2019). Isso indica um investimento da ordem de 1 bilhão de euros em cada recanto da EU na próxima década, para a construção do desenvolvimento sustentável no continente europeu e seus países membros. São metas bem claras, em linguagem simples e de aspirações comuns a todos, porém de forte impacto positivo em termos ambientais, por exemplo - "que a Europa lidere a questão dos plásticos de utilização única, pois os dez plásticos que se encontram em maior frequência nas praias da EU já são objeto da legislação europeia." (von der Leyen, 2019).

Os EUA disputam as primeiras posições em vários desses mercados, destacando-se no de Energia Elétrica, P\&D e Tecnologia da Informação. Já em maio de 2014, o Governo Americano reunia no encontro regional para a Parceria de Manufatura Avançada - AMP 2.0, os principais atores do ecossistema, constituído por membros da cúpula industrial, de universidades e do governo federal, para discutir e propor caminhos para garantir a liderança dos EUA em tecnologias emergentes, geradoras de empregos novos na manufatura de alta qualidade, capaz de potencializar a competitividade global do país americano (Kagermann e Wahister, 2013).

Encontros como aqueles impulsionaram o surgimento de provedores de avanços inovadores, em busca do melhor desenvolvimento ambiental, social e econômico, e motivaram os já existentes a romperem fronteiras e buscar o que há de melhor no mundo para os EUA, a exemplo do Instituto de Tecnologia de Massachusetts (MIT), fundado para acelerar a revolução industrial do país. Os graduados daquela Universidade inventaram tecnologias fundamentais, lançaram novas indústrias e criaram milhões de empregos para os americanos. Atrai talentos do mundo inteiro, através do ensino, da pesquisa e da inovação. Os novos caminhos para a inovação em 2021 abordam a tecnologia antirracista nos EUA, inclusão digital, salas de aula equitativas, segurança em saúde e pandemias e ecossistemas resilientes, cujo programa foi batizado como MIT Solve 2021 Global Challenges, lançado em março desse mesmo ano. A oferta é de mais de 1,5 milhões de dólares em prêmios de financiamento para projetos inovadores, oriundos de qualquer parte do mundo. São embaixadores Solve pessoas ilustres como o CEO da Zoom - Eric S. Yuan, e Karlie Kloss, fundador da Kode With Kloss. Este programa tem por financiadores e parceiros: fundações, empresas e organizações do setor público e privado (MIT, 2021).

No caso do Brasil, o Programa das Nações Unidas para o Desenvolvimento (PNUD, 2020), lançou em 2020 o Projeto de Formação e Treinamento de Gestores Públicos para a Implementação de Políticas Públicas na Agenda 2030, em parceria com a Escola Nacional de Administração Pública (ENAP) e com o Banco Nacional de Desenvolvimento Econômico e Social (BNDES), denominado de Plataforma Desafios, Faculdade Latino-Americana de Ciências Sociais (FLACSO), Banco Interamericano de Desenvolvimento (BID), Banco de Desenvolvimento da América Latina (CAF), Tribunal de Contas da União (TCU), BB Seguros, BB Consórcios, Cielo, Cateno, Livelo, Banco do Brasil e Caixa Econômica Federal, com o objetivo de incentivar e apoiar a sociedade para combater os impactos da COVID-19 e minimizar seus efeitos, através do uso das tecnologias. (PNUD, 2020).

No sentido de promover a participação da juventude, outras parcerias foram constituídas pelo PNUD, e lançada uma chamada pública com o nome de Jogo Digital Cidade do Amanhã, visando ao empoderamento dos jovens na criação de um jogo inédito sobre os Objetivos do Desenvolvimento Sustentável 16 - Paz, Justiça e Instituições Eficazes. Um dos programas chama-se Inovar o IDH - Atlas de Desenvolvimento Humano. Na versão de 2020, a plataforma ganhou uma melhoria tecnológica que é capaz de realizar

Rev. Bras. Gest. Amb. Sustent., 2021, vol. 8, n. 19, p. 1203-1217. 
uma aproximação indicativa com os Objetivos de Desenvolvimento Sustentável (ODS), por meio de correlação entre o Perfil de Desenvolvimento e a Agenda 2030 (PNUD, 2020).

Nesse contexto de abrangência mundial, tanto as políticas públicas advindas dos governos, como as ações de empresas e organizações estão trilhando o caminho efetivo para o desenvolvimento de práticas de gestão mais responsáveis. Uma possibilidade real para atingir esse importante objetivo é a perseguição por indicadores, que de fato qualifiquem essas ações, que sejam acessíveis a todos e possam ser traduzidos em certificações que confirmem o compromisso do setor público, do setor privado e do terceiro setor com o desenvolvimento sustentável, objetivo maior caracterizado neste presente estudo. Diante de tão relevante tema e de valores significativos de ordem socioeconômica e ambiental, este artigo tem por objetivo identificar a evolução das medidas de sustentabilidade e as consequentes certificações, e como tal combinação corrobora para o desenvolvimento sustentável, caracterizando as contribuições dadas pelo segmento produtivo e governamental.

\section{Histórico das certificações}

A origem das certificações ambientais tem como vetor precursor as ações de responsabilidade social no mundo corporativo (Garcia, 2017). Iniciou-se de forma mais efetiva ao longo das décadas de 1950 e 1960, nos Estados Unidos, podendo ser considerada um marco conceitual da responsabilidade social.

Outro marco destacável na direção de uma visão progressiva da necessidade de pensar estrategicamente em sustentabilidade foi a revolução industrial, a partir do século XIX, cujas transformações naturais causadas pelo homem provocaram impactos sociais e econômicos. Um tema ainda embrionário sob a ótica daquela época de acordo com Conto et al. (2017), porém, as mudanças profundas nas relações socioeconômicas, provocadas pela revolução industrial, propiciaram a base para a introdução da temática ambiental.

A Organização Mundial das Nações Unidas, em sua comissão sobre o meio ambiente, apresentou o relatório Brundtland (1987), que se tornou um marco quanto ao conceito de sustentabilidade e definiu o desenvolvimento sustentável como sendo aquele que "satisfaz as necessidades do presente sem comprometer a capacidade de as futuras gerações satisfazerem as suas próprias necessidades" (Rodrigues et al., 2015).

Em 2012 o Brasil sedia a conferência, a Rio+20 no Rio de Janeiro. Baseada em três pilares, ou triple bottom line: econômico, social e ambiental. Destacam-se os temas "Economia Verde e sua Contribuição para a Erradicação da Pobreza", e a "Estrutura da Governança para o Desenvolvimento Sustentável no Âmbito das Nações Unidas" (Brasil, 2016).

0 monitoramento histórico do desempenho ambiental é uma ferramenta que permite que as organizações inspecionem e supervisione os impactos ambientais de suas operações, através dos dados ambientais ao longo do tempo. Os registros devem conter os parâmetros mais importantes, tais como: emissões, resíduos, efluentes, materiais, uso de energia e água, eficiência, produção, demanda, acidentes e multas ambientais. Essas informações devem ser analisadas a fim de encontrar o máximo de impactos ambientais importantes da organização e fazer propostas para reduzir ou minimizá-los em uma estrutura de melhoria contínua (Campos e Melo, 2008).

\section{Certificações de sustentabilidade}

De uma maneira geral, nas informações levantadas, a maioria das fontes trazem certificações relacionadas a setores de atividades específicos, referentes à construção civil, agricultura e manejo florestal, produtos e serviços etc. Por exemplo, Thomé (2016) identificou (i) Selo Azul da Caixa Econômica Federal, uma classificação socioambiental para os projetos habitacionais financiados pela própria Caixa; (ii) Certificação Leed, um sistema internacional de certificação orientação ambiental para edificações, utilizado em 
143 países; (iii) Certificação AQUA-HQE, certificação internacional dirigida às construções sustentáveis, desenvolvida a partir da certificação francesa Démarche HQE (Haute Qualité Environmentale), aplicada no Brasil pela Fundação Vanzolini (Thomé, 2016).

Uma das certificações de grande utilização é a série ISO 14000 e as suas derivações. Ela corresponde a um Sistema de Gestão Ambiental criado pela International Organization for Standardization (ISO), apresentando diretrizes para auditoria ambiental, avaliação do desempenho ambiental, rotulagem ambiental e análise do ciclo de vida do produto. Permite que ao implementá-las, as organizações formulem políticas e diretrizes de acordo com as normas e legislação ambiental, revisitando seus valores, princípios e sistemas operacionais para cumprimento de uma estratégia minimizadora de impactos ambientais negativos. Em 1993, através da criação do Comitê Técnico - TC-207, composto por trinta diferentes países, dentre eles o Brasil, foram elaboradas uma série de normas referentes ao sistema de gestão ambiental, tais como ISO 14001 (que possui certificação), ISO 14004, ISO 14010 e ISO 14011, traduzidas para aplicação no Brasil pela Associação Brasileira de Normas Técnicas (ABNT), validadas a partir do final de 1996. Enquanto na Europa, cerca de 3000 empresas já possuíam esta certificação no final da década de 1990, o Brasil alcançou a número de 88 empresas certificadas com a ISO 14000, em 1999. Além das normas citadas acima, compõem o ambiente da série ISO 14000, as normas ISO 14020, ISSO 14031, ISO 14040 e o guia ISO 64 (Silva et al., 2020).

De acordo com a pesquisa anual de certificados válidos para as normas ISO, realizada em 2018, o Brasil caiu para a 14aㅡ colocação, com 1,44\% de empresas certificadas em relação ao mundo, enquanto a China ocupou a primeira colocação, detendo 30,71\% das certificações mundiais da ISO 14001 (Inntelectus, 2018).

\section{Indicadores de sustentabilidade}

No artigo publicado por Conto et al. (2017), é possível verificar que a maioria dos indicadores utilizados para a concessão de certificados ambientais na construção civil, consideram as variáveis referentes à geração de resíduos sólidos, líquidos e gasosos por serem um dos principais fatores a serem discutidos em todo o mundo, conforme citado por Azzi et al. (2015). Ressalva-se ainda, que a cadeia de geração de resíduos inclui a extração, processamento, transporte e eliminação (Russel-Smith e Lepech, 2015).

Desta forma, procura-se estabelecer os critérios de avaliação, identificando-se primeiro quais são as dimensões a serem avaliadas e a partir daí cria-se os indicadores.

Ainda de acordo com Conto et al. (2017), no Brasil, dentre as certificações mais utilizadas estão a LEED (Leadership in Energy \& Environmental Design), AQUA (Alta Qualidade Ambiental), Procel Edifica e o selo Casa Azul Caixa.

No sistema LEED as dimensões avaliadas são: implantação sustentável, eficiência hídrica, energia e atmosfera, materiais e recursos, conforto ambiental, inovação e projeto e por fim, créditos regionais. A quantidade de pontos obtidos pode variar entre 40 (nível certificado) a 110 (nível platina) (Chang e Tsai, 2015).

A metodologia do sistema AQUA leva em consideração duas dimensões: o sistema de gestão ambiental e a qualidade ambiental da edificação (Oliveira, 2014).

O selo Procel objetiva a redução de consumo de energia elétrica e estabelece cinco níveis de qualificação: A B, C, D e E (PROCEL, 2016).

O selo Casa Azul Caixa possui critérios de avaliação em seis dimensões: qualidade urbana, projeto e conforto, eficiência energética, conservação de recursos materiais, gestão da água e práticas sociais (Conto et al., 2017).

De acordo com Lieshout et al. (2008), a convergência das tecnologias propiciará, também, uma menor intensidade de carga ao meio ambiente, também denominada de pegada ecológica.

Mais recentemente é possível perceber a realização de trabalhos diversos fazendo correlações entre indicadores de sustentabilidade e fatores expoentes da tecnologia digital 
e da indústria 4.0, indicando caminhos para o aperfeiçoamento de tais práticas, a exemplo do que propõem Enyoghasi e Badurdeen (2021), quando elaboram estudos viabilizando a análise dos 6 Rs - Reutilizar, Reduzir, Redesenhar, Reciclar, Recuperar e Remanufaturar com a sua aplicabilidade em relação a produtos, processos e sistemas manufaturados.

\section{Materiais e métodos}

A metodologia utilizada nesta pesquisa é a abordagem qualitativa, a qual possibilita a análise e interpretação por parte do pesquisador, com suas apreciações sobre o fenômeno em estudo, conforme definido por Pereira et al. (2018). 0 estudo também se valeu da pesquisa exploratória, com o objetivo de investigar um problema, para fornecer informações mais precisas, bem como, permitir maior proximidade com o tema, de acordo com Gil (2007). O estudo usou a técnica indicada por Bardin (1977) para a análise de corpus de texto.

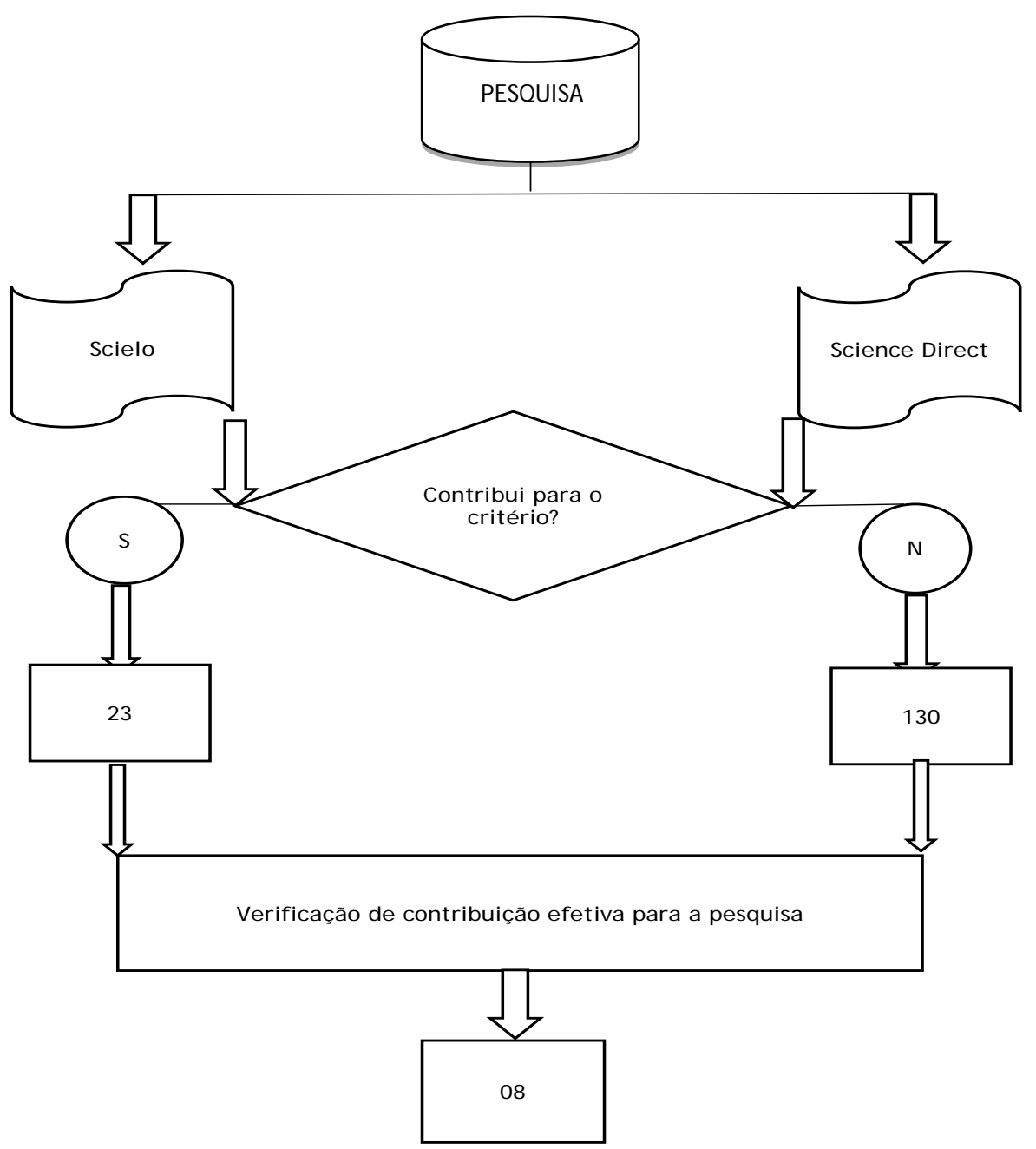

Figura 1. Sequenciamento da pesquisa e número de achados. 
Dessa forma, foi realizado o levantamento de informações no ambiente do Periódicos CAPES e nas seguintes bases científicas: Scielo e Science Direct. Foram utilizados os termos de busca: Medidas de Sustentabilidade nas Organizações; Certificações de Sustentabilidade Ambiental existentes no Mundo; Quantos e Quais são os Certificados de Sustentabilidade Ambiental no Brasil; Inovação em "Selos Verdes".

A Figura 1 descreve o esforço sequencial de pesquisa, achados e aproveitamento de trabalhos importantes para o embasamento teórico e para a percepção ora delineada.

De acordo com Bardin (1977), em estudos de caráter qualitativo é possível se utilizar da técnica de análise de conteúdo, para se efetuar a realização do levantamento de dados, organizá-los, descrevê-los, definir critérios de escolha e de apresentação de resultados sobre o tema pesquisado. Trata-se, portanto, de uma técnica que requer esforço de interpretação por parte do pesquisador, como acrescenta Creswell (2007). Apesar da existência atual de softwares de análise bibliométrica, eficazes para a composição de análise estatística de conteúdos, este artigo, por se tratar de trabalho com caráter de aprendizagem, levou o pesquisador a optar pela realização da denominada "leitura flutuante" indicada por Bardin (2006), cuja forma permite a leitura dinâmica por parte do pesquisador em relação aos 153 trabalhados achados pelo critério de busca definido e, assim, organizá-los e escolhê-los, de acordo com o nível de aproximação de seu conteúdo com o objetivo do trabalho, valendo-se da interpretação prevista como técnica efetiva em estudos qualitativos. Essa escolha, apesar de caráter subjetivo, propiciou maior conhecimento pela leitura dos artigos encontrados e pautou-se por critérios objetivos definidos da seguinte forma: I. 0 estudo possui caráter abrangente, em vez de específico, sobre indicadores e certificações sustentáveis? II. Trata das questões relacionadas à importância de indicadores para as certificações? III. Observa causas ou consequências para a humanidade em função da sustentabilidade?

A resposta esperada para efetuar as escolhas seria: sim. Não obstante a ocorrência de estudos que não responderam positivamente às questões acima, a leitura flutuante de tais estudos, permitiu o aproveitamento parcial de algumas informações que serviram para subsidiar a formação da linha de abordagem do presente artigo, conforme as diversas citações realizadas e a lista de referências.

No processo de busca foram encontrados 153 trabalhos que tinham relação com os temas, porém apenas 23 contribuíam diretamente para os critérios. Apesar disso, vários aspectos dos 153 achados foram aproveitados para compor a base teórica deste artigo. $\mathrm{Na}$ análise aprofundada daqueles 23 trabalhos, 08 obras foram escolhidas em função de sua maior relevância para esta pesquisa e dos critérios adotados, sob a ótica subjetiva do pesquisador, objeto da iniciativa acadêmica deste artigo, reiteradamente um processo de aprendizagem. Por isso, se justifica os estudos que aqui são destacados, considerando a necessidade e importância de que outros estudos sejam desenvolvidos para complementar os parâmetros e resultados expostos neste trabalho.

\section{Resultados e discussão}

De acordo com Conto et al. (2017), cresce cada vez mais a adesão e o desenvolvimento de selos e certificações ambientais, como meio de mitigar as ações e os impactos ambientais. Caracterizando, as necessidades de planejamento estratégico de longo prazo, diante do cenário de competitividade internacional, fundamentais na proveniência de resultados, com base no compromisso de medidas de sustentabilidade, através das certificações.

No sentido de potencializar a importância da visão comum entre cidades e organizações em relação à sustentabilidade e o desenvolvimento no cenário mundial, os autores Kitchin et al. (2015), apresentam a ideia de que "os indicadores requisitam de uma 
metodologia que favoreça a troca de informações entre regiões, como forma de se maximizar o alcance de objetivos necessários à sustentabilidade."

Já Enyoghasi e Badurdeen (2021), afirmam que a manufatura abrange "três elementos fundamentais para obtenção de resultados em sustentabilidade: produtos, processos e sistemas." Os autores indicam a existência de oportunidades em se alcançar a fabricação sustentável, "através de clusters de métricas para a avaliação de produtos, processos e sistemas", com a utilização simultânea das novas tecnologias disponíveis, associadas aos parâmetros dos "6Rs - reduzir, recuperar, reutilizar, redesenhar, remanufaturar e reciclar".

Esses três estudos citados acima, são formas exemplares dos achados da pesquisa deste artigo, salientando desde já a importância de se obter estudos complementares. Além deles, as contribuições de outros autores para o entendimento do papel das certificações para a melhoria da relação da sociedade com o uso dos recursos naturais, estão destacados na Tabela 1, em função dos critérios adotados.

Tabela 1. Contribuições de autores sobre o papel das certificações.

\begin{tabular}{|l|l|}
\hline \multicolumn{1}{|c|}{ Autor } & \multicolumn{1}{|c|}{ Principais contribuições } \\
\hline Kalafatis et al. (1999) & $\begin{array}{l}\text { Propicia uma visão da aprendizagem histórica da evolução das relações } \\
\text { de consumo es fatores que aceleram a mudança de comportamento, } \\
\text { rumo à sustentabilidade. }\end{array}$ \\
\hline Tayra e Ribeiro (2006) & $\begin{array}{l}\text { Indicadores de primeira, segunda e terceira geração, nesta última se } \\
\text { encontram aqueles que são vinculantes, sinérgicos e transversais, que } \\
\text { simultaneamente incorporam atributos do Desenvolvimento Sustentável, } \\
\text { diferente dos primórdios que consistiam em listas estanques de } \\
\text { indicadores sem integração. }\end{array}$ \\
\hline OECD (2007). & $\begin{array}{l}\text { Harmonização das iniciativas no campo dos indicadores ambientais, } \\
\text { buscando o desenvolvimento de um conjunto para uso internacional. }\end{array}$ \\
\hline Antón e Díaz (2014) & $\begin{array}{l}\text { Aplicação holística dos métodos BIM e LCA, que focam respectivamente } \\
\text { gerenciar informações no ciclo de vida do projeto e avaliar o } \\
\text { desempenho ambiental. Ambos visando à sustentabilidade e ao } \\
\text { equilíbrio entre critérios ambientais, sociais e econômicos. }\end{array}$ \\
\hline Roa e Vargas (2016). & $\begin{array}{l}\text { Elucida a relação do ser humano com o meio ambiente, demonstrando } \\
\text { como os organismos vivos se modificam durante a troca e interação com } \\
\text { o meio externo e suas consequências para a saúde. }\end{array}$ \\
\hline
\end{tabular}

Pela descrição dos artigos aqui citados, e pelos tópicos citados na Tabela 1, na coluna sobre as principais contribuições, de acordo com seus respectivos autores, identifica-se a busca por estudos na direção de otimizar as relações entre tecnologias e indicadores, para a melhor qualidade de vida e das organizações, de uma forma abrangente e universal. Portanto, reunir, sistematizar, arquivar, gerar séries históricas, comparar, avaliar, controlar e planejar, são fatores preponderantes para tomar decisões. Esse é um processo complexo e um desafio a ser enfrentado pelos que querem fazer uso da sustentabilidade para propiciar melhorias organizacionais, sociais e econômicas em prol do desenvolvimento sustentável. 


\section{Considerações finais}

A partir dos artigos analisados para o presente estudo, foi possível constatar uma relação dialética entre causas e consequências, como as aqui citadas:

- o mundo vem enfrentando nas últimas décadas uma profunda transformação social, econômica e ambiental;

- para lidar com tais transformações, se faz necessário educar as pessoas e qualificar as organizações para o enfrentamento correto das mudanças;

- esta qualificação passa por produtos, serviços, processos, comunicação, estruturas e pessoas, estejam elas no setor público ou privado;

- as mudanças têm por paradigma a aplicabilidade da estratégia de intervenção, a qual perpassa o correto uso dos instrumentos da administração e do planejamento estratégico, alicerces maiores para a obtenção de resultados no curto, médio e longo prazos;

- os avanços tecnológicos são fatores decisivos para enfrentar o desafio, cada vez maior, da escassez de recursos, frente à crescente demanda da sociedade por produtos, serviços e oportunidades;

- para a obtenção do equilíbrio entre esses fatores, recursos e demandas, os indicadores e certificações de sustentabilidade podem fazer a diferença entre o possível e o necessário para o justo desenvolvimento da humanidade.

Diante dos achados referentes às certificações existentes e às medidas adotadas como dimensões para análise de sustentabilidade e a respectiva definição de seus indicadores, verifica-se uma lacuna para que se constitua um parâmetro único de comparação entre organizações, empresas e regiões. As dificuldades passam por problemas relacionados à falta de uniformidade dos critérios de análise adotados por diversas certificações, base de dados disforme em função do setor de atividade, do tamanho, da região e da série histórica disponível.

$\mathrm{Na}$ atualidade, existem muitas iniciativas de construção de indicadores de sustentabilidade, mas "em sua maioria as variáveis não se encontram necessariamente integradas e ordenadas", a não ser para responder temas bem específicos, assim é o que citam Tayra e Ribeiro (2006). De acordo com esses autores, pode-se classificar os indicadores como de primeira, segunda e terceira geração, nesta última se encontram a transversalidade, o efeito vinculante e a sinergia em prol da sustentabilidade.

Em todo o mundo, há um "claro processo de desenvolvimento conceitual, metodológico e instrumental. As experiências compreendem tentativas que utilizam escalas diversas" (nacional, estadual, municipal...) e enfoques bastante diferentes entre si (Quiroga-Martinez, 2003).

"As características socioambientais aferidas na pesquisa evidenciaram exigências de sustentabilidade diferenciadas para cada tipo de empresa. Os resultados da análise destes fatores de influência pesquisados, permitiram a identificação de características socioambientais intrínsecas a cada tipo de organização" (Tachizawa e Pozo, 2010).

"Um indicador deve ser entendido como um parâmetro ou valor derivado de parâmetro, que aponta e fornece informações sobre o estado de um fenômeno com uma extensão significativa" (OECD, 1993).

Van Bellen (2002) apresenta alguns conceitos sobre indicadores, entre eles, o de que é uma medida que resume informações relevantes sobre um fenômeno em particular ou um substituto desta medida. Este conceito caracteriza a essência da enorme contribuição que as organizações e empresas, notadamente as que compõem o cenário do avanço tecnológico mundial, estão dando para a maximização do uso das informações, a minimização de riscos, a previsão e projeção de resultados, a economia de recursos, de

Rev. Bras. Gest. Amb. Sustent., 2021, vol. 8, n. 19, p. 1203-1217. 
espaço e de tempo, tudo isso em consonância ao progresso sustentável, cada vez mais exigido pela sociedade mundial.

Percebe-se, ainda, que há um grande desafio a ser enfrentado em relação à unicidade de sistemas, base de dados, parâmetros comuns e padronizáveis, perspectiva de comparabilidade criteriosa e científica entre organizações, empresas e regiões. Porém, os avanços tecnológicos atuais, possuem instrumentos que geram a possibilidade de reunir grandes quantidades de informações, sistematizá-las, criar modelos comparativos e permitirem facilidade de acesso com economia de tempo e de recursos.

Então, a crescente exigência por celeridade na melhoria de procedimentos sustentáveis, implica na efetividade das soluções possíveis de se viabilizar. Esta evolução é percebida pelo aumento da consciência coletiva do consumidor sobre a importância do meio ambiente, a maior atenção da mídia para esta temática, o fortalecimento dos grupos defensores da causa ambiental e das legislações mais rigorosas para se evitar grandes desastres ecológicos. Daí o surgimento das estratégias de negócios focadas em sustentabilidade e "a importância em se combater as ideologias falseadas na oferta desvirtuada de produtos e de marcas hipoteticamente defensoras da sustentabilidade" (Kalafatis et al., 1999).

Certamente, este é um trabalho que pode ser aperfeiçoado em relação à abrangência da busca por informações estruturadas sobre o tema abordado e, com maior profundidade, em relação aos critérios de análise das certificações e indicadores de sustentabilidade. Porém, aqui não se pretendeu esgotar o assunto e sim, provocar um melhor entendimento sobre tema de tamanha importância, notadamente nos tempos atuais, onde o mundo constata cada vez maiores e mais frequentes acidentes e fenômenos extremos da natureza, com impactos ambientais, sociais e econômicos cada vez mais danosos aos seres vivos de nosso planeta, porém, com enormes oportunidades tecnológicas para realizar com maestria esse enfrentamento.

\section{Conflito de interesses}

Os autores declaram não haver conflito de interesses.

\section{Referências}

Antón, L. Á.; Díaz, J. Integration of life cycle assessment in a BIM environment. Procedia Enginnering, v. 85, p. 26-32, 2014. https://doi.org/10.1016/j.proeng.2014.10.525

Azzi, M.; Duc, H.; Ha, Q. P. Toward sustainable energy usage in the power generation and construction sectors: A case study of Australia. Automation in Construction, v. 59, p. 122-127, 2015. https://doi.org/10.1016/j.autcon.2015.08.001

Bardin, L. Análise de conteúdo. Lisboa: Edições 70, 2006.

Bardin, L. L'ére logique. Paris: Robert Laffont, 1977.

Brasil. Ministério do Meio Ambiente. Agenda 21 Global. Brasília: MMA, 2016. Disponível em: <http://www.mma.gov.br/responsabilidade-socioambiental/agenda-21/agenda21global>. Acesso em: 05 jan. 2016.

Brundtland, G. Our common future: call for action. Environmental Conservation, v. 14, n. 4, p. 291-294, 1987. https://doi.org/10.1017/S0376892900016805

Campos, L. M. S.; Melo, D. A. Indicadores de desempenho dos Sistemas de Gestão Ambiental (SGA): uma pesquisa teórica. Produção, v. 18, n. 3, p. 540-555, 2008. https://doi.org/10.1590/S0103-65132008000300010 
Chang, A. S.; Tsai, C. Y. Sustainable design indicators: Roadway project as an example. Ecological Indicators, v. 53, p.137-143, 2015. https://doi.org/10.1016/ j.ecolind.2015.01.036

China Hoje. A política chinesa para o desenvolvimento sustentável. 2021. Disponível em: <http://www.chinahoje.net/a-politica-chinesa-para-o-desenvolvimento-sustentavel/>. Acesso em: 01 mar. 2021.

Conto, V.; Oliveira, M. L.; Ruppenthal, J. E. Certificações ambientais: contribuição à sustentabilidade na construção civil no Brasil. Gestão da Produção, Operações e Sistemas, v. 12, n. 4, p. 100-127, 2017. https://doi.org/10.15675/gepros.v12i4.1749

Creswell, J. W. Projeto de pesquisa: métodos qualitativo, quantitativo e misto. Porto Alegre: Artmed, 2007.

Dalmoro, M.; Venturini, J. C.; Pereira, B. A. D. Marketing verde: responsabilidade social e ambiental integradas na envolvente de marketing. Revista Brasileira de Gestão de Negócios, v. 11, n. 30, p. 38-52, 2009.

Embalagem Marca. Pesquisa: consumidores exigem embalagens de roupas mais sustentáveis no e-commerce. 2020. Disponível em: <https://www.embalagemmarca. com.br/2020/09/pesquisa-consumidores-exigem-embalagens-de-roupas-mais-

sustentaveis-no-e-commerce/>. Acesso em: 28 fev. 2021.

Enyoghasi, C.; Badurdeen, F. Industry 4.0 for sustainable manufacturing: Opportunities at the product, process, and system levels. Resources, Conservation \& Recycling, v. 166, 105362, 2021. https://doi.org/10.1016/j.resconrec.2020.105362

Frezatti, F.; Relvas, T.; Nascimento, A.; Junqueira, E.; Bido, D. Perfil de planejamento e ciclo de vida organizacional nas empresas brasileiras. Revista de Administração, v. 45, n. 4, p. 383-399, 2010. https://doi.org/10.1016/S0080-2107(16)30469-1

Garcia, T. Como surgiu a responsabilidade social. 2017. Disponível em: <https://www.dialogusconsultoria.com.br/como-surgiu-a-responsabilidade-social/>.

Acesso em: 10 jul. 2020.

Gil, A. C. Como elaborar projetos de pesquisa. 4. ed. São Paulo: Atlas, 2007.

Inntelectus. Pesquisa anual de empresas certificadas ISO no Brasil. 2018. Disponível em: <http://blog.inntelectus.com.br/pesquisa-anual-de-empresas-certificadas-iso-no-brasil/>. Acesso em 13 mar. 2021.

Kagermann, H.; Wahister, W. Recommendations for implementing the strategic initiative Industrie 4.0. Acatech - National Academy of Science and Engineering, 2013. https://doi.org/10.1108/07363769910289550

Kalafatis, S. P.; Pollard, M.; East, R.; Tsogas, M. H. Green marketing and Ajzen's Theory of Planned Behaviour: A cross-market examination. Journal of Consumer Marketing, v. 16, n. 5, p. 441-460, 1999. https://doi.org/10.1108/07363769910289550

Kappa, S. Pesquisa sobre comportamento do consumidor E-Commerce. 2020. Disponível em: <https://www.smurfitkappa.com/br/newsroom/2020/consumers-making-a-fashionstatement-when-it-comes-to-sustainability>. Acesso em: 18 jan. 2021.

Kitchin, R.; Lauriault, T. P.; McArdle, G. Knowing and governing cities through urban indicators, city benchmarking and real-time dashboards. Regional Studies, Regional Science, v. 2, n. 1, p. 6-28, 2015. https://doi.org/10.1080/21681376.2014.983149 
Lester, D.; Parnell, J. A.; Carraher, S. Organizational life cycle: A five stage empirical scale. The International Journal of Organizational Analysis, v. 11, n. 4, p. 339-354, 2003. https://doi.org/10.1108/EB028979

Lieshout, M.; Enzing, C.; Hoffknecht, A.; Holtmannspötter, D.; Noyons, E.; Companò, R.; Butter, R.; van der Giessen, A.; Bodea, G.; Malanowski, N. Converging applications enabling the Information Society. Düsseldorf: Zukünftige Technologien Consulting, 2008. (Technical report).

MIT - Massachusetts Institute of Technology. MIT Solve announces 2021 global challenges. 2021. Disponível em: <https://news.mit.edu/2021/mit-solve-announces-2021-globalchallenges-0302>. Acesso em: 02 mar. 2021.

Newtrade. Consumidores pagarão em média 35\% a mais por produtos sustentáveis e de procedência transparente em 2020. 2020. Disponível em: <https://newtrade.com.br/economia/consumidores-pagarao-em-media-35-a-mais-porprodutos-sustentaveis-e-de-procedencia-transparente-em-2020/>. Acesso em: 28 fev. 2021.

OECD - Organization for Economic Co-Operation and Development. Core set of indicators for environmental performance reviews: A synthesis report by the group on the state of the environment. Paris: OECD, 1993. (Environment monographs, 83).

OECD - Organization for Economic Co-Operation and Development. Indicators of sustainable development: Guidelines and methodologies. 3. ed. New York: OECD, 2007.

Oliveira, V. M. Sistemas de certificação ambiental e norma brasileira de desempenho. Juiz de Fora: Universidade Federal de Juiz de Fora, 2014. (Dissertação de mestrado).

OMS - Organização Mundial da Saúde. Informe sobre la salud en el mundo: forjemos el futuro, 2003. Disponível em: <https://apps.who.int/iris/handle/10665/42822>. Acesso em: 17 jan. 2021.

OMS - Organización Mundial de la Salud. Ambientes saludables para los niños. OMS, 2002. Disponible en: <http://www.who.int/world-health-day/previous/2003/backgrounder /es>. Acesso em: 24 nov. 2020.

Pereira, A. S.; Shitsuka, D. M.; Parreira, F. J.; Shitsuka, R. Metodologia da pesquisa científica. Santa Maria: UAB/NTE/UFSM, 2018.

PNUD - Programa das Nações Unidas para o Desenvolvimento. Disrupção digital e inovação. Brasília: PNUD Brasil, 2020.

PNUD - Programa das Nações Unidas para o Desenvolvimento. Guia PCS - Produção e Consumo Sustentáveis. Brasília: PNUD Brasil, 2016.

PROCEL - Programa Nacional de Eficiência Energética em Edificações. Centro Brasileiro de Informações de Eficiência Energética. 2016.

Quiroga-Martinez, R. Los indicadores de desarrollo sostenible: estado del arte. Santiago do Chile: CEPAL, 2003. (Curso-Taller Indicadores de Desarrollo Sostenible para América Latina y el Caribe).

Roa, L. A.; Vargas, B. P. La salud del ser humano y su armonía con el ambiente. Revista Med, v. 24, n. 1, p. 111-122, 2016. https://doi.org/10.18359/rmed.2338

Silva, A. R.; Ohara, L. F.; Ghizzi, M. L. P. Normas ISO 14000: sistema de gestão ambiental. 2020. Disponível em: <http://www.qualidade.esalq.usp.br/fase2/iso14000.htm>. Acesso em: 13 mar. 2021. 
Tachizawa, T.; Pozo, H. Monitoramento do Passivo Socioambiental com o Suporte da Tecnologia de Informação. Journal of Information Systems and Technology

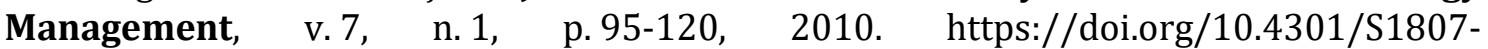
17752010000100004

Tatsachen Ueber Deutschland. Sustentabilidade e meio ambiente. 2021. Disponível em: $<$ https://www.tatsachen-ueber-deutschland.de/pt-br/sustentabilidade-e-meioambiente>. Acesso em: 01 mar. 2021.

Tayra, F.; Ribeiro, H. Modelos de indicadores de sustentabilidade: síntese e avaliação crítica das principais experiências. Saúde e Sociedade, v. 15, n. 1, p. 84-95, 2006. https://doi.org/10.1590/S0104-12902006000100009

Thomé, B. B. 5 selos de sustentabilidade que agregam valor às suas obras. SIENGE, 2016. Disponível em: <https://www.sienge.com.br/blog/selos-de-sustentabilidade-agregandovalor-as-suas-obras/>. Acesso em: 18 jan. 2021.

Van Bellen, H. M. Indicadores de sustentabilidade: uma análise comparativa. Florianópolis: Universidade Federal de Santa Catarina, 2002. (Tese de doutorado).

von der Leyen, U. A Union that strives for more: My agenda for Europe. 2019. (Political Guidelines for the Next European Commission.). Disponível em: $<$ https://ec.europa.eu/info/sites/default/files/political-guidelines-nextcommission_en_0.pdf>. Acesso em: 02 mar. 2021. 